\title{
Anthropometric differences in the tibial tuberosity to trochlear groove measurement in an African population
}

\author{
Rankin $\mathrm{M}^{1} \stackrel{\mathbb{D}}{ }$, Mohideen $\mathrm{MAF}^{2}$ \\ 1 MBChB(UKZN), FC Orth (SA); Registrar, Department of Orthopaedics, School of Clinical Medicine, University of KwaZulu-Natal \\ BSc(UKZN), MBChB(Medunsa), FC Orth(SA); Orthopaedic Consultant, Westville Life Hospital
}

Corresponding author: Dr Mario Rankin, Nelson R Mandela School of Medicine UKZN, Department of Orthopaedics, 719 Umbilo Road, Durban, 4001; email: mario.rankin@gmail.com; cell: +27731421476

\begin{abstract}
Background: The tibial tuberosity to trochlear groove (TTTG) is a well-described radiographic measurement around the knee, which is of clinical significance in the assessment of patients with patellar instability. The TTTG is clinically relevant when considering the type of surgical procedures that may be required. The purpose of this retrospective observational study was to measure the TTTG measurement in the black African population and to compare it to the quoted norms in the literature.

Methods: A random sample was obtained of 100 consecutive CT scans of lower limbs for vascular pathologies from black African patients. The TTTG was measured by a single observer on the Siemens ${ }^{\circledR}$ syngo.plaza software.

Results: CT scans of 162 knees from 88 patients met the inclusion criteria. The median age was 34 years (range 19-85 years; interquartile range [IQR] 24-50). The majority of cases were male $(75 \% ; n=121)$. The mean TTTG was $20.3 \mathrm{~mm}$ (standard deviation [SD] 16.1; range 1-29.2 mm). The mean TTTG in males was $20.7 \mathrm{~mm}$ (95\% confidence interval [Cl] 19.9-21.5 mm) and $19.1 \mathrm{~mm}(95 \%$ $\mathrm{Cl} 17.6-20.5 \mathrm{~mm})$ in females $(p=0.06)$. There was no correlation found between age and TTTG $(p=0.12)$.

Conclusion: According to our study, barring some limitations, it appears that there may be a noteworthy difference in the TTTG measurement in the black African population. Larger comparative studies would be required to confirm these findings and to determine the clinical relevance.
\end{abstract}

Level of evidence: Level 4

Key words: tibial tuberosity trochlear groove distance, measurement, anatomical variation, ethnic differences

Citation: Rankin M, Mohideen MAF. Anthropometric differences in the tibial tuberosity to trochlear groove measurement in an African population. SA Orthop J 2019;18(3):53-57. http://dx.doi.org/10.17159/2309-8309/2019/v18n3a6

Editor: $\operatorname{Dr}$ C Snyckers, University of Pretoria, Pretoria, South Africa

Received: January 2019

Accepted: May 2019

Published: August 2019

Copyright: () 2019 Rankin M, et al. This is an open-access article distributed under the terms of the Creative Commons Attribution Licence, which permits unrestricted use, distribution and reproduction in any medium, provided the original author and source are credited.

Funding: No funding was received for this study.

Conflict of interest: The authors declare they have no conflicts of interest that are directly or indirectly related to the research. 


\section{Introduction}

The tibial tuberosity to trochlear groove (TTTG) measurement was first described by Goutallier and Bernageau in 1978 as a radiographic measurement on X-rays. The measurement was described on the AP X-ray with the knee in $30^{\circ}$ flexion and neutral rotation, measuring the horizontal distance between two vertical parallel lines passing through the summit of the tibial tuberosity and the bottom of the trochlear groove. ${ }^{1}$ This measurement is of clinical significance in the assessment of patella instability. ${ }^{2}$ It plays a pivotal role when deciding on the need for surgery. ${ }^{2}$

The TTTG has been widely researched in the Western world using various modalities of imaging such as X-rays, computerised tomography (CT) scans and magnetic resonance imaging (MRI). ${ }^{3-5}$ In most studies, the normal TTTG measurement is less than $15 \mathrm{~mm} .^{6-10}$ The literature also suggests that in cases with a TTTG above $20 \mathrm{~mm}$, a bony surgical procedure (e.g. medialising osteotomy of tibial tuberosity) should be considered in addition to the soft tissue realignment or stabilising procedures. ${ }^{6,11,12}$ The original studies on the TTTG were, however, performed among the French population. There is no published data on the TTTG distance in the African population. This is relevant because several studies have been done in recent years that have shown how the human skeleton and orthopaedic measurements may vary across different ethnic groups. Koerner et al. found differences in the femoral version of the African American population. ${ }^{13}$ Igbigbi et al. did a study on the tibio-femoral angle of the Malawian population and the results showed ethnic differences from Caucasian values. ${ }^{14}$ Hence this may have importance with regard to the threshold for choice of surgical intervention and could possibly make way for improved protocols encompassing ethnic differences.

The purpose of this retrospective observational study was to measure the TTTG measurement in the black African population and compare it to the quoted norms in the literature.

\section{Methods}

This was a retrospective observational study by means of a chart review. A random sample of 100 consecutive cases were identified from a database

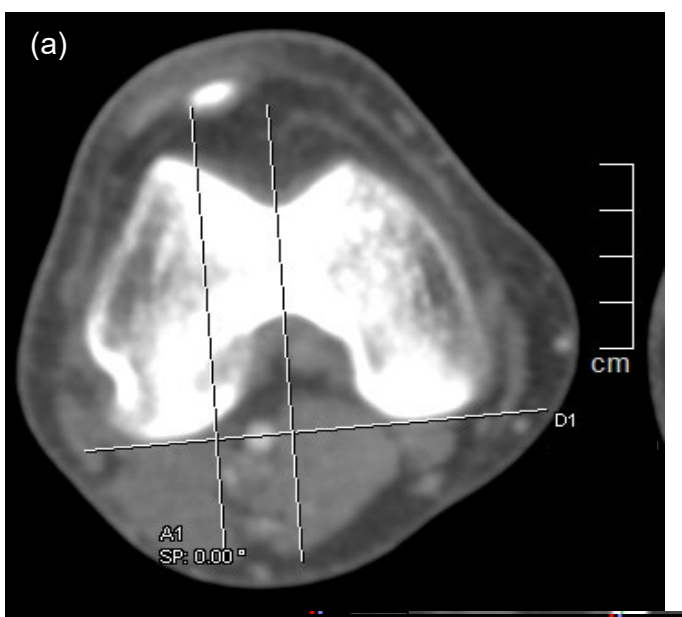

Figure 1. CT scan slices illustrating a sample measurement in a case with a normal TTTG measurement. (a) The axial slice of the initial reference line perpendicular to the posterior condylar line and through the deepest part of the trochlear groove is illustrated; (b) The line parallel to the reference line through the summit of the tibial tuberosity is shown; the TTTG measurement line (red dotted line measurement $1.50 \mathrm{~cm}$ ) perpendicular to both yellow dotted lines is also shown; (c) The navigated sagittal slice confirming the summit of the tibial tuberosity
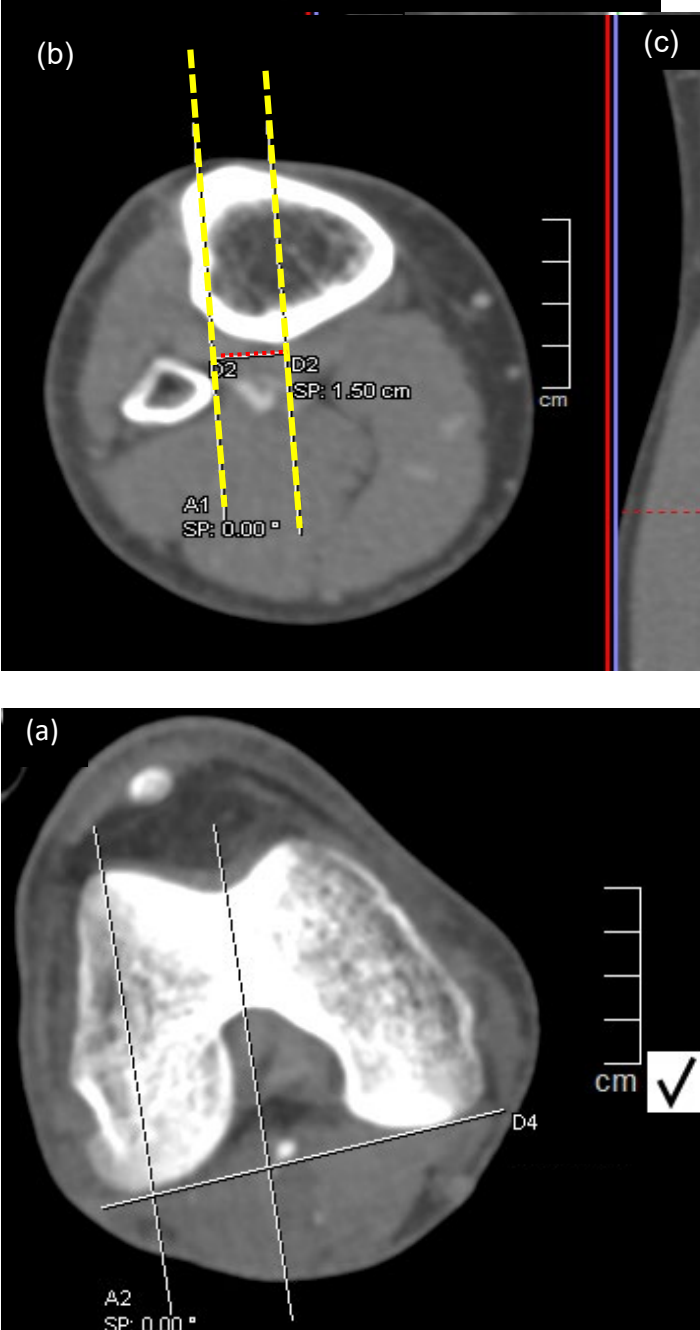

(b)

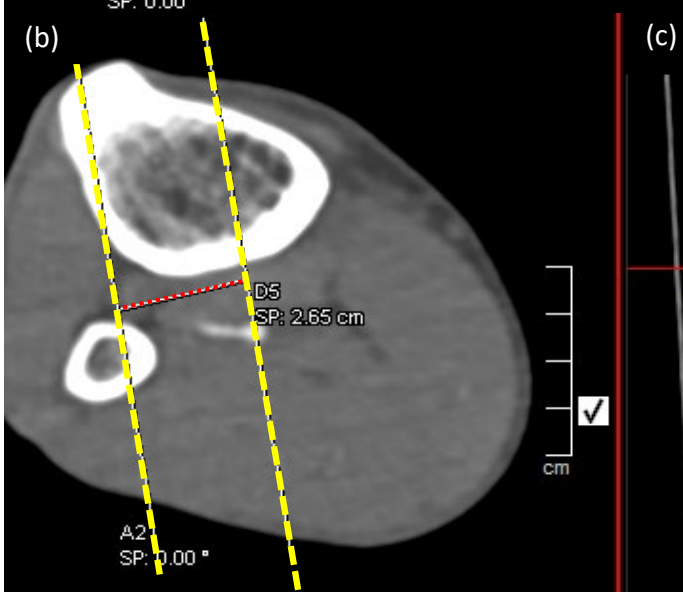

(c)
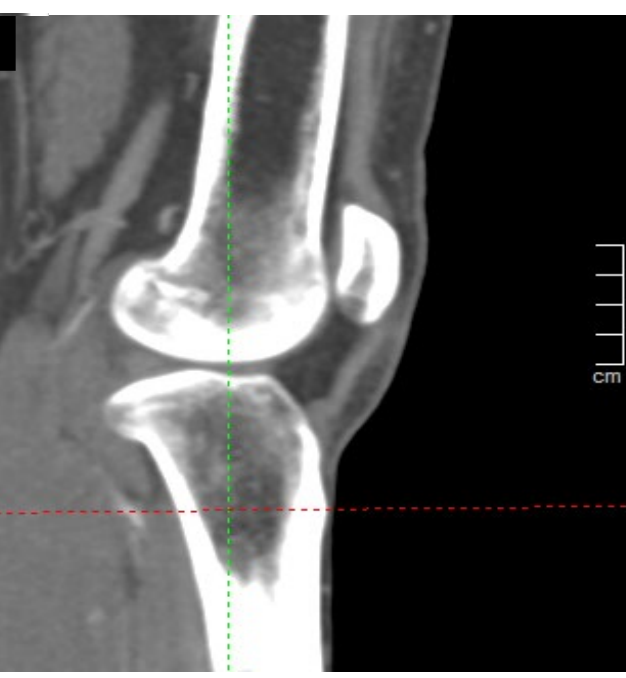

Figure 2. CT scan slices illustrating a sample measurement in a case with an abnormal TTTG measurement. (a) The axial slice of the initial reference line perpendicular to the posterior condylar line and through the deepest part of the trochlear groove; (b) The line parallel to the reference line through the summit of the tibial tuberosity, as well as the TTTG measurement line (red dotted line measurement $2.65 \mathrm{~cm}$ ) perpendicular to both yellow dotted lines; (c) The navigated sagittal slice confirming the summit of the tibial tuberosity 
at a tertiary level hospital as patients referred from the vascular surgery department to radiology for CT angiograms between years 2013 and 2018. These were patients admitted to the vascular department predominantly for elective atraumatic vascular pathologies (e.g. peripheral vascular disease, vascular stab injuries, etc.), and hence were considered asymptomatic from an orthopaedic perspective. The ethnic classification of patients as black Africans was self-reported by patients and recorded during administrative registration of the patient on the hospital information system. Exclusion criteria included: knee dislocations, periarticular fractures, gross arthritic changes of the knee and previous bony knee surgery.

The TTTG was measured using the syngo.plaza software (Siemens ${ }^{\circledR}$, Munich, Germany). The actual TTTG measurement was performed as described by Dejour et al. ${ }^{8}$ using the axial cuts of the CT scan of the knee joints, with the navigation tool of the sagittal cuts used to improve landmark accuracy (Figure 1). Patients were positioned supine with the knees in extension. A tangential line was drawn along the posterior femoral condyles on the axial cuts. A perpendicular line was then drawn in the AP plane on the axial cut at the centre and the most anterior point of the tibial tuberosity. A parallel line was drawn through the deepest point of the trochlear groove. These points were confirmed by utilising the sagittal cuts in the navigation window to improve accuracy of identifying the anatomical landmark. A measurement was then obtained by measuring the distance between the two parallel lines in the AP plane. This was done in the left and the right knees of all cases that met the patient selection criteria.

Statistical analysis was performed using Stata 15.0 (StataCorp. College Station, Texas). Continuous variables were reported as mean $( \pm S D$ ) or median (with interquartile range) and categorical variables as number and percentages, unless otherwise stated. Differences in TTTG by sex were compared using the unpaired t-test. Spearman correlation was used to correlate TTTG by age. All tests were two-sided and the level of significance was set at $\mathrm{p}<0.05$.

\section{Results}

CT scans of 100 patients were viewed. A total of 88 patients met the inclusion criteria. Thirty-eight knees were excluded from the analysis: 12 had severe OA, 11 had significant trauma, 12 scans were not peripheral enough to measure appropriately, two knees were in excessive flexion, and one patient had an above-knee amputation of one leg. This left 162 knees on which measurement and analysis was performed (Figure 2).

The median age was 34 years (range 19-85 years; interquartile range $[I Q R] 24-50)$. The majority of cases were male $(75 \% ; n=121)$. The mean TTTG was $20.3 \mathrm{~mm}$ (standard deviation [SD] 16.1; range 1-29.2 mm) (Figure 3). While the TTTG was slightly larger in males than females, the difference was not significant in this cohort. The mean TTTG in males was $20.7 \mathrm{~mm}$ (95\% confidence interval [Cl] $19.9-21.5 \mathrm{~mm})$ and $19.1 \mathrm{~mm}(95 \% \mathrm{Cl} 17.6-20.5 \mathrm{~mm})$ in females $(p=0.06)$. There was no correlation found between age and TTTG $(p=0.12)$.

\section{Discussion}

The aim of this study was to measure the TTTG measurement in the black African population and compare it to the quoted norms in the literature. We found that the mean TTTG was $20.3 \mathrm{~mm}$. The difference between sexes was not significant in this cohort. There was also no correlation found between age and TTTG. In most studies the normal TTTG measurement is less than $15 \mathrm{~mm}^{6-10}$ These original studies were performed by Dejour et

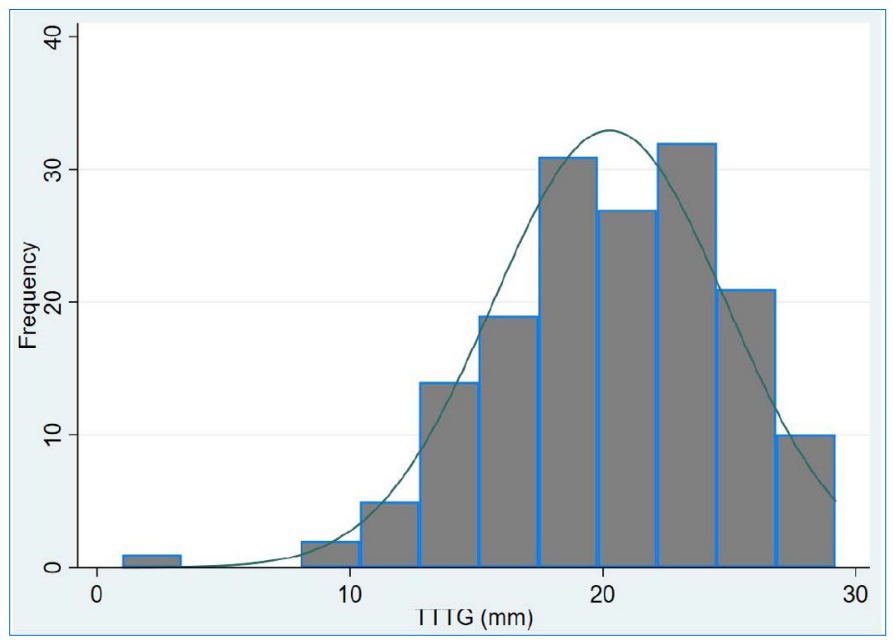

Figure 3. The mean tibial tuberosity to trochlear groove (TTTG) measurement was found to be $20.3 \mathrm{~mm}(\mathrm{n}=162)$

al. among the French population. ${ }^{8}$ There is no published data on the TTTG distance in the African population. Researchers from China, Korea and India have also undertaken studies to compare the differences in their respective populations with regard to TTTG measurements.

They all found differences in the average TTTG distance among their populations when compared to the Western quoted norm measurements. The study performed in Korea yielded a mean TTTG measurement of $10.24 \mathrm{~mm} .{ }^{15}$ In this study they also measured the TTTG on CT scans of 100 patients (50 of each sex); however, only 85 of them were asymptomatic patients meeting the inclusion criteria. The mean age in this study was 55 years (range 25-82 years). Another strong point about this study was that measurements were done in a blinded manner by three separate observers, on two occasions. Kulkarni et al. concluded that the TTTG distance in the Indian population, using MRI as the measurement modality, is significantly different when compared to the published Western data. ${ }^{16} \mathrm{~A}$ mean of $13.5 \mathrm{~mm}$, with a range of 7.3-19.8 mm, was reported in a sample size of 100 knees. The mean age in this sample was 37 years (range 20-61 years). In the Chinese population, a smaller average measurement was found in asymptomatic individuals used as a control group, with mean of $10.1 \mathrm{~mm}$ in a sample size of 73 control knees. ${ }^{17}$ The mean age in this cohort was 27 years (range 16-38 years). This age group is more appropriate for the concerned pathology which is usually present in adolescents and young adults. ${ }^{18,19}$

One of the possible reasons for varying measurement results between studies may potentially be explained by the ethnic differences. In a systematic review, Kim et al., also highlighted morphological differences that exist in certain anatomical features and measurements around the knee. ${ }^{20}$ Koerner et al. have also questioned the definition of 'normal' femoral versions among sexes and ethnicities. ${ }^{13}$ Our data brings about the discussion of possible ethnic differences when it comes to the TTTG measurement. Furthermore, the clinical implication of such a finding remains unclear. Tse et al., for example, suggested that the threshold for tibial tuberosity transfer should be lower in Chinese patients due to these differences. ${ }^{17}$

Some points for discussion may be raised regarding limitations to this study. Although the sample size for this study was relatively small ( $n=162$ knees), it did exceed the required sample size of 68 , with a chosen confidence level (CL) constant at 95\%, an acceptable margin of error (MOE) of $10 \%$ and an SD of 0.5 . Previous international studies of this nature utilised comparable sample sizes of between 73 and $100 . .^{10,15-17}$ The consideration of inter-observer reliability with regard to performing the actual measurement should be taken into 
account when evaluating the strength of this study. ${ }^{21-23}$ In this study only a single individual (a senior orthopaedic registrar) performed the measurements for data collection. To improve the accuracy of average measurements, as with any study of this nature, it would be beneficial to have more than one person performing measurements. The exact degree of knee flexion was not measured in this study. To our knowledge, at the time of this study, only six publications were noted to have assessed the TTTG through different ranges of knee flexion. ${ }^{24-29}$ Dietrich et al. reported that the TTTG increases significantly at the end stage of extension of the knee due to a biomechanical action known as the 'screw-home mechanism'. ${ }^{25}$ Carlson et al. also showed that a static TTTG measurement cannot accurately predict dynamic lateral displacement of the patella. ${ }^{26}$ In our study, the CT scans were performed with the patients lying supine with knees in extension. Current literature mentions that the routine CT scan should be taken with the knee in full extension. 8,30 It does not, however, specify the range of knee flexion at which the CT scan can be deemed acceptable for TTTG measurement. We do know, however, that most patella instability occurs from $0^{\circ}$ to $30^{\circ}$ of knee flexion. ${ }^{29} \mathrm{CT}$ slice thickness of $3 \mathrm{~mm}$ was used in the majority of cases. This in itself can play a role in determining the landmarks for measurement. ${ }^{22}$ Accuracy may be improved with a smaller slice thickness. Although all of the patients in this study were self-reported as black African by race, the results may not necessarily be generalisable. A much larger sample from different regions throughout Africa will be required for this purpose. Some might argue that the assumption that given that these patients were chosen from the vascular surgery department does not mean that they are completely asymptomatic for patella-femoral instability. This is a valid point because no patients were screened for signs and symptoms of patella instability prior to CT scanning.

Future research on this topic will help verify this data. Ideally a larger comparative series involving symptomatic and asymptomatic individuals of various ethnicities is required to determine clinically relevant reference values. Accuracy of data can be improved by involving multiple blinded experienced observers with low thickness CT slices and consistent knee flexion angles.

\section{Conclusion}

According to our study, barring some limitations, it appears that there may be a difference in the TTTG measurement in the asymptomatic black African population when comparing it to data published elsewhere. This may be of significance with regard to the threshold for choice of surgical intervention. Larger comparative studies would be required to confirm these findings and to determine the clinical relevance.

\section{Ethics statement}

All procedures performed in studies involving human participants were in accordance with the ethical standards of the institutional and/or national research committee (reference number BE631/16) and with the 1964 Helsinki declaration and its later amendments or comparable ethical standards. For this study formal consent was not required.

\section{Declaration}

The authors declare authorship of this article and that they have followed sound scientific research practice. This research is original and does not transgress plagiarism policies.

\section{Author contributions}

MR contributed to the original study concept, proposal write-up, data collection and analysis, and final article write-up. MAHM contributed to the study design, layout and final article concepts.

\section{ORCID}

Rankin M (D) http://orcid.org/0000-0001-5247-4062 Mohideen MAH iD http://orcid.org/0000-0003-1205-0702

\section{References}

1. Goutallier D, Bernageau J, Lecudonnec B. [The measurement of the tibial tuberosity. Patella groove distanced technique and results (author's transl)]. Revue de chirurgie orthopedique et reparatrice de l'appareil moteur 1978;64(5):423-28.

2. Liu JN, Steinhaus ME, Kalbian IL, et al. Patellar instability management: a survey of the International Patellofemoral Study Group. Am J Sports Med 2017:363546517732045. doi: 10.1177/0363546517732045

3. Schoettle PB, Zanetti M, Seifert B, et al. The tibial tuberositytrochlear groove distance; a comparative study between CT and MRI scanning. The Knee 2006;13(1):26-31. doi: 10.1016/j. knee.2005.06.003

4. Thakkar RS, Del Grande F, Wadhwa V, et al. Patellar instability: $\mathrm{CT}$ and MRI measurements and their correlation with internal derangement findings. Knee surgery, sports traumatology, arthroscopy: official journal of the ESSKA 2015 doi: 10.1007/ s00167-015-3614-8

5. Ho CP, James EW, Surowiec RK, et al. Systematic techniquedependent differences in CT versus MRI measurement of the tibial tubercle-trochlear groove distance. Am J Sports Med 2015;43(3):675-82. doi: 10.1177/0363546514563690

6. Grawe B, Stein BE. Tibial Tubercle Osteotomy: Indication and Techniques. J Knee Surg 2015;28(4):279-84. doi: 10.1055/s-0035-1544973

7. Alemparte J, Ekdahl M, Burnier L, et al. Patellofemoral evaluation with radiographs and computed tomography scans in 60 knees of asymptomatic subjects. Arthroscopy : the journal of arthroscopic \& related surgery: official publication of the Arthroscopy Association of North America and the International Arthroscopy Association 2007;23(2):170-77. doi: 10.1016/j.arthro.2006.08.022

8. Dejour H, Walch $\mathrm{G}$, Nove-Josserand L, et al. Factors of patellar instability: an anatomic radiographic study. Knee surgery, sports traumatology, arthroscopy : official journal of the ESSKA 1994;2(1):19-26.

9. Ding DY, Kanevsky R, Strauss EJ, et al. Anteromedialisation tibial tubercle osteotomy for recurrent patellar instability in young active patients: A retrospective case series. Injury 2016;47(3):737-41. doi: 10.1016/j.injury.2015.10.005

10. Pandit S, Frampton C, Stoddart J, et al. Magnetic resonance imaging assessment of tibial tuberosity-trochlear groove distance: normal values for males and females. International Orthopaedics 2011;35(12):1799-803. doi: 10.1007/s00264-011-1240-8

11. Frosch $S$, Balcarek $P$, Walde TA, et al. [The treatment of patellar dislocation: a systematic review]. Zeitschrift fur Orthopadie und Unfallchirurgie 2011;149(6):630-45. doi: 10.1055/s-0030-1250691

12. Mulford JS, Wakeley CJ, Eldridge JD. Assessment and management of chronic patellofemoral instability. J Bone Joint Surg Br 2007;89(6):709-16. doi: 10.1302/0301-620X.89B6.19064

13. Koerner JD, Patel NM, Yoon RS, et al. Femoral version of the general population: does 'normal' vary by gender or ethnicity? Journal of orthopaedic trauma 2013;27(6):308-11. doi: 10.1097/ BOT.0b013e3182693fdd

14. Igbigbi PS, Msamati BC. Tibiofemoral angle in Malawians. Clinical anatomy 2002;15(4):293-6. doi: 10.1002/ca.10024

15. Song EK, Seon JK, Kim MC, et al. Radiologic measurement of tibial tuberosity-trochlear groove (TT-TG) distance by lower extremity rotational profile computed tomography in Koreans. Clinics in orthopedic surgery 2016;8(1):45-8. doi: 10.4055/cios.2016.8.1.45

16. Kulkarni S, Shetty AP, Alva KK, et al. Patellar instability in the Indian population: relevance of tibial tuberosity and trochlear groove distance. Sicot-J 2016;2:14. doi: 10.1051/sicotj/2016008

17. Tse MS, Lie CW, Pan NY, et al. Tibial tuberosity-trochlear groove distance in Chinese patients with or without recurrent patellar dislocation. Journal of orthopaedic surgery 2015;23(2):180-81.

18. Atkin DM, Fithian DC, Marangi KS, et al. Characteristics of patients with primary acute lateral patellar dislocation and their recovery within the first 6 months of injury. Am J Sports Med 2000;28(4):472-79. doi: 10.1177/03635465000280040601

19. Fithian DC, Paxton EW, Stone ML, et al. Epidemiology and natural history of acute patellar dislocation. Am J Sports Med 2004;32(5):1114-21. doi: 10.1177/0363546503260788

20. Kim TK, Phillips $M$, Bhandari $M$, et al. What differences in morphologic features of the knee exist among patients of 
various races? A systematic review. Clin Orthop Relat Res 2017;475(1):170-82. doi: 10.1007/s11999-016-5097-4

21. Dornacher D, Reichel H, Lippacher S. Measurement of tibial tuberosity-trochlear groove distance: evaluation of inter- and intraobserver correlation dependent on the severity of trochlear dysplasia. Knee surgery, sports traumatology, arthroscopy: official journal of the ESSKA 2014;22(10):2382-87. doi: 10.1007/ s00167-014-3083-5

22. Koeter S, Horstmann WG, Wagenaar FC, et al. A new CT scan method for measuring the tibial tubercle trochlear groove distance in patellar instability. The Knee 2007;14(2):128-32. doi: 10.1016/j. knee.2006.11.003

23. Miles JE, Jensen BR, Kirpensteijn $\mathrm{J}$, et al. Measurement repeatability of tibial tuberosity-trochlear groove offset distance in red fox (Vulpes vulpes) cadavers. American journal of veterinary research 2013;74(6):888-94. doi: 10.2460/ajvr.74.6.888

24. Izadpanah K, Weitzel E, Vicari M, et al. Influence of knee flexion angle and weight bearing on the tibial tuberosity-trochlear groove (TTTG) distance for evaluation of patellofemoral alignment. Knee surgery, sports traumatology, arthroscopy: official journal of the ESSKA 2014;22(11):2655-61. doi: 10.1007/s00167-013-2537-5

25. Dietrich TJ, Betz M, Pfirrmann CW, et al. End-stage extension of the knee and its influence on tibial tuberosity-trochlear groove distance (TTTG) in asymptomatic volunteers. Knee surgery, sports traumatology, arthroscopy: official journal of the ESSKA 2014;22(1):214-18. doi: 10.1007/s00167-012-2357-z

26. Carlson VR, Sheehan FT, Shen A, et al. The relationship of static tibial tubercle-trochlear groove measurement and dynamic patellar tracking. Am J Sports Med 2017;45(8):1856-63. doi: 10.1177/0363546517700119

27. Marquez-Lara A, Andersen J, Lenchik L, et al. Variability in patellofemoral alignment measurements on MRI: influence of knee position. AJR Am J Roentgenol 2017;208(5):1097-102. doi: 10.2214/AJR.16.17007

28. Tanaka MJ, Elias JJ, Williams AA, et al. Correlation between changes in tibial tuberosity-trochlear groove distance and patellar position during active knee extension on dynamic kinematic computed tomographic imaging. Arthroscopy : the journal of arthroscopic \& related surgery: official publication of the Arthroscopy Association of North America and the International Arthroscopy Association 2015;31(9):1748-55. doi: 10.1016/j. arthro.2015.03.015

29. Seitlinger G, Scheurecker G, Hogler R, et al. The position of the tibia tubercle in 0 degrees to 90 degrees flexion: comparing patients with patella dislocation to healthy volunteers. Knee surgery, sports traumatology, arthroscopy: official journal of the ESSKA 2014;22(10):2396-400. doi: 10.1007/s00167-014-3173-4

30. Servien E, Verdonk PC, Neyret P. Tibial tuberosity transfer for episodic patellar dislocation. Sports Med Arthrosc Rev 2007;15(2):61-67. doi: 10.1097/JSA.0b013e3180479464 